\title{
Functional Disorders of Heart Stimulating Systems in Patients Treated with Radiation Therapy - Own Experiences
}

\author{
Ewa Ziółkowska ${ }^{1}$, Tomasz Wiśniewski ${ }^{2}$, Mieszko Białas ${ }^{3}$ \\ ${ }^{1,2,3}$ Department of Radiotherapy, Prof. F. Łukaszczyk Memorial Oncology Center, Bydgoszcz, Poland
}

\begin{abstract}
Objectives: To analyze our experiences in irradiation of patients with implanted pacemakers and to draft guidelines regarding the therapy with ionizing radiation in this group. Methods: We developed an algorithm of management of patients with implanted heart stimulator who were qualified to radiotherapy. These rules were applied to 50 patients treated at our center in 2009 2012. Results: A total of 55 radiation therapy procedures were performed amongst 50 studied patients, including 31 (56\%) radical radiotherapies and 24 (44\%) palliative radiotherapies. The majority of heart stimulating systems in our group were pacemakers (47 radiation therapy procedures, $85 \%)$; seven (13\%) patients were implanted with cardioverter-defibrillator, and one (2\%) had cardiac resynchronization device. In the majority of cases, the heart stimulating system was located outside the irradiated area (23 procedures, 42\%). However, the stimulator was placed in the therapeutic area in 10 (18\%) patients, and one of them required displacement of the device to the right infraclavicular area. In another 22 individuals (40\%) the stimulator was implanted in close proximity to the irradiated area. Mean dose received by a pulse generator was equal to $1.43 \mathrm{~Gy}$ (range 0-3 Gy). Only three patients (5.5\%) showed transient irradiation-related functional defects of heart stimulating system. Conclusions: Irradiation of patients with heart stimulator is rarely associated with the functional disorders of the stimulating system. The presence of heart stimulating system does not constitute an absolute contraindication to radiation therapy. There is a need of tight cooperation between radiotherapist and cardiologist in the course of radiotherapeutic process.
\end{abstract}

Keywords: radiation therapy, stimulator, cardioverter-defibrillator

\section{Introduction}

The number of oncological patients who possess heart stimulating system and are treated with radiotherapy has dynamically increased during the recent years. Present evidence suggests that the use of irradiation in patients with implanted heart stimulating systems represents significant diagnostic and therapeutic challenge in oncological practice. This results from two reasons. Firstly, dynamic progress in advanced technologies involved in therapeutic devices for malignant neoplasms has taken place, and innovative techniques of radiotherapy, which can negatively affect the heart stimulating system, were implemented. Secondly, constant modification of implantable electrotherapeutic devices, including the use of a new generation of implantable cardioverters-defibrillators (ICD), was reflected by considerable increase in the susceptibility of this equipment to ionizing radiation.

Patients subjected to radiotherapy are exposed to two types of radiation. Nonionizing electromagnetic radiation, i.e. a dispersed disturbance of electromagnetic field in the form of a transverse wave, whose electric and magnetic component are perpendicular to each other and transform each other. The radiation originates from oscillating or accelerated electrons; in the case of accelerators, klystron or magnetron (i.e. devices responsible for acceleration of electron) represent the main sources of radiation. The intensity of nonionizing radiation is the highest during switching on and off of the linear accelerator. Heart stimulator can record the disturbance of electromagnetic field as a myocardial potential, which can be reflected by disorders of detection, exit block, stimulation with constant rhythm, or, in extremely rare cases, reprogramming of the device. Most of these disorders are transient in nature and resolve spontaneously after switching off of the electromagnetic field $[1,2]$. Moreover, they can be prevented by proper programming of the stimulator. The structure of presently used modern linear accelerators was improved during the recent years, leading to marked reduction in the emission of electromagnetic radiation. Therefore, it has been proposed that the effects of this radiation do not need to be considered as a potential cause of malfunction of heart stimulator [3]. The situation is markedly different in the case of cardioverters, which are approximately 5-10 times more susceptible to the influence of external electromagnetic field [4]. Therefore, it is recommended to switch off the antiarrhythmic function of cardioverter prior to radiation therapy session in order to prevent an inadvertent jolt during the treatment $[5,6]$.

Ionizing radiation interacts with material, which results in the generation of ions. The influence of ionizing radiation on the function of stimulators is hard to assess. Probably, the malfunction results from ionization of metal elements included in the electronic systems. Newer generations of pacemakers have more complex structure and more fragile electronic systems, and therefore are more susceptible to ionizing radiation. Their components are made of silicone, which loses electrons from its covalence shells when exposed to ionizing radiation; this may cause the malfunction of the whole electronic system. Although the most frequently observed disorders include changes in the frequency of work, also the problems with device's memory were documented, manifesting as reprogramming or reset of predefined parameters (especially in the case of cardioverters-defibrillators) or even complete switching off [7]. Contrary to nonionizing electromagnetic radiation, the 


\section{International Journal of Science and Research (IJSR) \\ ISSN (Online): 2319-7064 \\ Index Copernicus Value (2013): 6.14 | Impact Factor (2014): 5.611}

effects of ionizing radiation are frequently irreversible and can accumulate. Therefore, according to all published guidelines, cardiac pacemaker should not be placed in the irradiated area $[5,8]$. However, it should be remembered that even if the stimulator is placed outside the irradiated area, it can be affected by scattered radiation or radiation leakage through the shields. This risk should be always considered during planning of the therapy $[9,10]$.

The aim of this paper is to present the results of prospective observational study analyzing the complications of radiotherapy in patients with heart stimulating systems.

\section{Material and Methods}

The study included 50 patients who were treated at the $1^{\text {st }}$ Department of Radiotherapy and Teleradiotherapy Subunit of Prof. F. Łukaszczyk Memorial Regional Comprehensive Cancer Center in Bydgoszcz between January $1^{\text {st }}, 2009$ and June $30^{\text {th }}, 2012$. A total of 55 radiation therapy procedures were performed in this group, including 31 (56\%) radical radiotherapies and $24(44 \%)$ palliative radiotherapies. Three patients received more than one course of irradiation; however, different body areas were irradiated in all three cases. Mean age of the studied group was 72.8 years (range 51-89). Male patients constituted $62 \%$ (31/50) of the group. The majority of heart stimulating systems in our group were pacemakers (47 radiation therapy procedures, 85\%), seven (13\%) patients were implanted with cardioverterdefibrillator, and one (2\%) had cardiac resynchronization device. Heart stimulating systems manufactured by Medtronic (55\%) were the most popular in our group, followed by those produced by Biotronik (25\%), and St. Jude Medical (20\%). Atrioventricular block was the most frequent reason for the implantation of the heart stimulating system $(42 \%)$, followed by sick sinus syndrome $(36 \%)$, atrial fibrillation $(9 \%)$, and ventricular flutter $(4 \%)$. The reasons for radiation therapy of our patients included breast cancer $(20 \%)$, prostate cancer $(20 \%)$, lung cancer $(16 \%)$, cancers of head and neck $(11 \%)$, colorectal cancer $(9 \%)$, lymphoma (7\%), renal cancer $(6 \%)$, and other malignancies (11\%)[Table 1]. The heart stimulating system was located outside the irradiated area in the majority of cases (23 procedures, $42 \%$ ). In the remaining cases, the stimulator was placed in the therapeutic area $(n=10,18 \%)$ or in its proximity $(n=22,40 \%)$. Mean dose received by the pulse generator was equal to 1.43 Gy (range 0-3 Gy)[Table2]

A type of cardiac pacemaker was determined in every patient (stimulator, cardioverter-defibrillator, or resynchronizer) due to various sensitivity of these devices to radiotherapy. Also a time and place where the implantation took place was determined, along with location of the device in relation to irradiated field. Detailed information on the device was obtained from a stimulator card which every patient is obliged to possess. Each patient was consulted by a cardiologist who examined status of the device and verified if cardiac activity was modulated by a stimulator. The necessity of switching off the antiarrhythmic function was considered in the case of persons with cardiovertersdefibrillators. During the next stage, a total dose of irradiation per device was calculated on the basis of treatment plan. Optimally, the planned dose did not exceed 2
Gy and 1 Gy for ICP and ICD, respectively. Heart rate and arterial blood pressure of each patient were determined prior to and after every session of radiotherapy Additionally, an electrocardiogram was obtained prior to the treatment in patients whose cardiac action was modulated by stimulator. The patients were introduced onto radiotherapeutic device in presence of radiotherapist in charge and resuscitation team (anesthetist and anesthesiology nurse). Cardiac activity was monitored during irradiation in order to detect any potential abnormalities of stimulator's function. The dose of irradiation received by the stimulator was determined during the first radiotherapeutic session and compared with the dose estimated prior to the treatment. Each patient was informed about the possibility of delayed radiotherapy-induced defect of the stimulator and the necessity of immediate referral in the case of any dysfunction of the device. Moreover, a visit at Cardiac Outpatient Clinic was scheduled 4-6 after completing the radiotherapy. The scheme of post-treatment controls followed the standard protocol for oncological patients.

In accordance with the adopted control criteria of adverse events, all patients were examined for the presence of the malfunction of heart stimulating system prior to and during radiation therapy. The pretreatment functional evaluation of the stimulator included the review of records in stimulator's card/book in order to identify the timing of malfunctions not related to oncological treatment. The identification of radiotherapy-induced malfunctioning of the stimulator was based on the following methods: 1) observation of typical clinical signs associated with the defect of stimulator on the basis of patient's medical history and radiotherapeutic documentation (irradiation card), 2) evaluation of the influence of radiotherapy on the function of heart stimulator based on the timing (day, hour) of disorders (review of data stored in the memory of the device) and electronic records of radiotherapeutic device documented in the irradiation card. Such control enabled determination of precise correlation between the timing of consecutive stages of radiotherapy and the occurrence of malfunction of heart stimulating system.

Data stored in the memory of the device was reviewed by means of telemetric communication via the application of the tip of the programmer to the pacemaker. Using a programmer which was appropriate for a given type of stimulator (consistent with its brand), a cardiologist read the current settings of the device, verified its predefined parameters, and reviewed data stored in its memory. The verification took place 1 to 6 months after completing radiotherapy, enabling detection of potential malfunction occurring both during the treatment and thereafter.

\section{Results}

Three functional defects of heart stimulating system were documented. In each case, the estimated dose per pulse generator was lower than 2 Gy.

\section{Case one}

A 79-year-old female patient with implantable cardiac pacemaker (ICP) was implanted the heart stimulator in DDD mode in 2009 due to arrhythmia in the form of grade III

\section{Volume 4 Issue 12, December 2015}




\section{International Journal of Science and Research (IJSR) \\ ISSN (Online): 2319-7064 \\ Index Copernicus Value (2013): 6.14 | Impact Factor (2014): 5.611}

atrioventricular block. In 2011, the patient underwent oncological treatment, including right side mastectomy with adjuvant chemotherapy due to the cancer of the right breast. In March 2012, the patient completed adjuvant radiation therapy comprised of 20 fractions of 2.25 Gy each at the right thoracic wall, up to the total dose of 45 Gy. Estimated dose per stimulator did not exceed 2 Gy. During the first session of radiotherapy, according to the hospitalimplemented procedure, the patient's heart function was monitored on a continuous basis under the supervision of radiotherapist and anesthesiologist.

Although the patient experienced multiple episodes of heart palpitations, vertigo, and headache as early as in the course of radiotherapy, she did not report them to radiotherapist in charge as she did associate them with the functioning of heart stimulator. Heart palpitations continued until the follow-up examination conducted within the framework of our study. In fact, patient's report of cardiologic problems was prompted by the information from the cardiologist on the necessity of changing the stimulation mode due to 36 episodes of atrial fibrillation recorded by the programmer. The patient declared the lack of similar signs prior to radiotherapy and chemotherapy.

The examination of heart stimulating system (records stored in its memory) conducted two months following irradiation revealed multiple episodes of atrial fibrillation during the third week of radiation therapy. Moreover, the readings of programmer confirmed switching the stimulation mode from DDD to DDI. The cardiologist reset the stimulation mode to its original value. Four weeks later, in the course of another follow-up visit, the patient declared being in a good condition and complete resolution of heart palpitations.

\section{Case two}

Post-irradiation malfunction of heart stimulating system was also observed in an 81-year-old male with prostate cancer, who was implanted with cardioverter-defibrillator in the left infraclavicular region five years earlier within the framework of secondary prevention of cardiac arrest. The patient was qualified to the radical radiotherapy in the pelvic area up to 74 Gy in 37 fractions using IMRT technique and $15 \mathrm{MV}$ energy beam. The cardioverter-defibrillator was placed outside the irradiated area, and the dose estimated during an in vivo dosimetry equaled 0 Gy. After the $17^{\text {th }}$ fraction, the patient reported sudden weakness and heart palpitations while being at home, which required urgent hospitalization in cardiologic department. According to the hospital documentation, the patient experienced complete reprogramming of the device with resultant ventricular tachycardia. After the reset of cardioverter parameters and pharmacological normalization of cardiac rhythm, the patient underwent the remaining 15 fractions of radiation therapy without any ICD defects. The programmer-based evaluation of the heart stimulating system, performed four weeks after completing the irradiation, did not show any functional disorders of the stimulator during the period following the defect mentioned above.

\section{Case three}

The third episode of radiotherapy-induced malfunction of heart stimulating system referred to a 75-yearl-old male with prostate cancer. In 2006, the patient was implanted with heart stimulator (St. Jude Medical) in a DDD stimulation mode due to sick sinus syndrome. The heart stimulating system was located outside the irradiated area, and the dose determined on the basis of an in vivo dosimetry equaled 0 Gy. Photon radiation with a nominal energy amounting 15 MV was used; the patient received total dose of 65 Gy in 25 fractions at the therapeutic area. During the radiotherapy, the patient did not report any problems related to the heart stimulating system and the treatment was continued without any breaks or complications. However, a programmer-based control of the stimulator performed four weeks after completing the irradiation revealed two episodes of supraventricular tachycardia lasting for a few seconds, both temporarily associated with the irradiation sessions.

\section{Discussion}

Available literature does not constitute a valuable source of information on adverse events associated with radiation therapy in patients with heart stimulating systems. We still lack reliable estimates on the number of complications observed in this group of patients. Case reports predominate in a group of in vivo studies. Comprehensive analysis of this evidence suggests that about $30 \%$ of these reports refer to radiotherapy-induced defects of the stimulating devices [11]. However, it should be assumed that the real fraction is lower as not every case of uncomplicated irradiation of patient with cardiac stimulator is reported in literature. We documented the malfunction of heart stimulating system in 3 out of 55 irradiated individuals with such devices, which corresponded to $5.5 \%$.

We documented three cases of heart stimulating system malfunction, the timing of which pointed to potential association with radiotherapy. The first of them was a 79year-old woman subjected to radical radiotherapy due to the cancer of right breast. A follow-up examination performed within two months after irradiation revealed that during the third week of radiotherapy the patient experienced multiple episodes of arrhythmia in the form of atrial fibrillation. Acceleration of the baseline rhythm of the stimulator was reflected by a reset of the device and switching the stimulation threshold from DDD to DDI. As widely known, at present all modern DDD pacemakers have a function of automated change of stimulation mode activated whenever supraventricular arrhythmia is detected (the so-called mode switching or mode conversion). The possibility of automatic mode conversion from DDD to DDI whenever atrial rhythm exceeds a predefined frequency allows for synchronous (atrioventricular) stimulation during arrhythmia-free periods. In contrast, during the episode of atrial fibrillation, the ventricular rhythm (in DDI mode) is controlled independently from the atrial rhythm, which prevents rapid ventricular stimulation [12]. Probably, this was the reason why our patient did not experience a serious discomfort and radiotherapy was not associated with evident clinical complications. An attempt to interpret the pathomechanism of hereby described malfunction of heart stimulator faces many difficulties. Firstly, there was no evidence for the defect of heart stimulating system; only the episode of arrhythmia concomitant to radiotherapy raised our suspicions. It should be noted, however, that the patient was implemented with stimulator three years earlier and since 


\section{International Journal of Science and Research (IJSR) \\ ISSN (Online): 2319-7064 \\ Index Copernicus Value (2013): 6.14 | Impact Factor (2014): 5.611}

then had not history of atrial fibrillation. Moreover, no episodes of fibrillation were documented during three months of post-radiotherapy follow-up. The arrhythmia could result from extensive stress associated with radiotherapeutic procedures. However, prior to irradiation the patient was subjected to surgery and intensive chemotherapy, both being equally potent stressors. Also the presence of electrodes in the heart is postulated a potential arythmogenic factor. Although the dose per impulse generator was lower than 2 Gy in our patient, the dose received by the electrodes exceeded $10 \mathrm{~Gy}$. The involvement of neutron radiation in the pathomechanism of interaction between the stimulator and accelerator is less likely as $6 \mathrm{MV}$ energy beams were used. The phenomenon of photodispersion, being a source of neutrons, is mostly associated with generation of energy beams higher than 10 MV. Electromagnetic interferences cannot be excluded as a cause of this dysfunction as they frequently cause defects of stimulators during radiation therapy sessions; usually, this malfunction is transient in character and resolves when irradiation is completed. Since our patient experienced problems also after completing the therapy, and ECG records (obtained during a follow-up visit after completing the therapy) showed the episodes of atrial fibrillation also during this period, another underlying mechanism of the complication should be considered. Namely, the involvement of scattered radiation in the development of the abovementioned disorder should be taken into account. Estimation of the dose on the basis of dose-volume histogram can lead to considerable error if the heart stimulator is placed at a large distance from the region exposed to high doses of radiation. This results from the fact that modern systems of treatment planning do not consider the involvement of scattered radiation in this region. Therefore, an in vivo dosimetry is widely recommended, at least during the first session of radiation therapy.

The second case of post-irradiation malfunction of cardiac pacemaker occurred in an 81-year-old patient with prostate cancer, who experienced ventricular tachycardia and ICD reset after the $17^{\text {th }}$ fraction of radiation therapy. His ICD was reset as a result of this harmful ventricular arrhythmia. This sudden and health threatening clinical situation required hospitalization in cardiologic ward and another resetting of cardioverter parameters. The dangerous complication occurred despite theoretically safe treatment plan (localization of the pacemaker outside the irradiated area, dose determined during in vivo dosimetry equal to $0 \mathrm{~Gy}$ ). Unnecessary discharges generated by ICD can induce lifethreatening ventricular flutter. One can assume the involvement of neutron radiation as a potential cause of the abovementioned disorders as irradiation energy $>10 \mathrm{MV}$ was used. The analysis of the published reports highlights marked increase in the incidence of heart stimulating system malfunction during the use of beams of photon radiation with energies exceeding $10 \mathrm{MV}$, even if the pacemakers were located at a large distance from the irradiated area. Potential effect of secondary neutrons generated by high energy within the tip of the device seems likely as heart stimulating systems are highly susceptible to the influence of corpuscular radiation. The results of ex vivo studies revealed that direct irradiation of pulse generator with a proton beam is always associated with a serious defect of the device.
Secondary neutrons constitute the main fraction of radiation generated during radiotherapy with a proton beam.

The third case of the radiotherapy-induced malfunction of heart stimulating system referred to a 75 -year-old patient with prostate cancer. He experienced two episodes of supraventricular tachycardia, both being in temporal association with the irradiation session. Similar to the first hereby described case, there was no unequivocal evidence for stimulator's defect, but only temporal relation between radiotherapeutic session and the episode of arrhythmia. Perhaps, the latter was associated with stress response. However, it could be also caused by disorders of electromagnetic field associated with switching on/off of the device can be assumed to constitute a potential cause of this phenomenon as the comparative analysis of data stored in the pacemaker's memory and that recorded on irradiation card showed that the episodes took place at the beginning of irradiation session. The nature of hereby mentioned complication is not completely understood. It is assumed that each model of heart stimulating system shows different susceptibility to electromagnetic disturbances generated by various devices, including linear accelerator [13]. Electromagnetic disturbances can lead to improper detection of myocardial potential, which in turn can cause an exit block, rigid rhythm of stimulation, or reprogramming of the device. Usually, these effects are transient and reversible [14].

\section{Conclusions}

1) As the irradiation of patients with heart stimulator is rarely associated with the functional disorders of the stimulating system, the presence of pacemaker should not limit the use of radiation therapy in oncological patients.

2) In view of potential life and health risks in patients with heart stimulating system who were qualified to radiotherapy, there is a need for recommendations regarding cooperation between radiotherapist and cardiologist.

3) We did not observe a relationship between the total and fractional dose received by heart stimulator and the prevalence of complications.

4) As the prevalence of functional disorders of heart stimulating system is higher in the case of using irradiation energy equal to $15 \mathrm{MV}$, radiation therapy with energies higher than $10 \mathrm{MV}$ should be avoided in patients with heart stimulating systems. 


\section{International Journal of Science and Research (IJSR) \\ ISSN (Online): 2319-7064 \\ Index Copernicus Value (2013): 6.14 | Impact Factor (2014): 5.611}

Table 1: Characteristics of medical procedures associated with radiation therapy in patients implemented with heart stimulating system.

\begin{tabular}{|l|l|}
\hline \multicolumn{1}{|c|}{ Parameter } & \multicolumn{1}{|c|}{$n(\%)$} \\
\hline Type of heart stimulating system & \\
- pacemaker: & $47(85 \%)$ \\
- DDD & $33(60 \%)$ \\
- AAI & $9(16 \%)$ \\
- VVI & $5(9 \%)$ \\
- cardioverter-defibrillator & $7(13 \%)$ \\
- resynchronizer & $1(2 \%)$ \\
\hline Manufacturer of heart stimulating system & \\
- Medtronic & $30(55 \%)$ \\
- Biotronik & $14(25 \%)$ \\
- St. Jude Medical & $11(20 \%)$ \\
\hline Heart condition & \\
- atrioventricular block & $23(42 \%)$ \\
- sick sinus syndrome & $20(36 \%)$ \\
- atrial fibrillation & $5(9 \%)$ \\
- ventricular flutter & $2(4 \%)$ \\
- other & $5(9 \%)$ \\
\hline Oncological diagnosis & \\
- breast cancer & $11(20 \%)$ \\
- prostate cancer & $11(20 \%)$ \\
- lung cancer & $9(16 \%)$ \\
- cancers of head and neck & $6(11 \%)$ \\
- colorectal cancer & $5(9 \%)$ \\
- lymphoma & $4(7 \%)$ \\
- kidney cancer & $3(6 \%)$ \\
- other malignancies & $6(11 \%)$ \\
\hline
\end{tabular}

Table 2: Doses received by pulse generator and electrodes of heart stimulating system

\begin{tabular}{|l|l|}
\hline \multicolumn{1}{|c|}{ Parameter } & \multicolumn{1}{|c|}{ Value } \\
\hline $\begin{array}{l}\text { Dose received by a generator }(G y) \\
\text { - mean (range) }\end{array}$ & $1.43(0-3)$ \\
$<2 \mathrm{~Gy}$ & $52(95 \%)$ \\
$>2 \mathrm{~Gy}$ & $3(5 \%)$ \\
$>10 \mathrm{~Gy}$ & 0 \\
\hline Dose received by electrodes $(G y)$ & \\
- mean (range) & $4.5(0-30)$ \\
$<2 \mathrm{~Gy}$ & $44(80 \%)$ \\
$>2 \mathrm{~Gy}$ & $8(15 \%)$ \\
$>10 \mathrm{~Gy}$ & $3(5 \%)$ \\
\hline
\end{tabular}

\section{Competing Interests}

The authors report no potential conflicts of interest.

\section{Funding}

This study received no special funding

\section{References}

[1] Cardall TY, Brady WJ, Chan TC, Perry JC, Vilke GM, Rosen P. Permanent cardiac pacemakers: issues relevant to the emergency physician, part II. J Emerg Med. 1999; 17: 697-709.

[2] Quertermous T, Megahy MS, Das Gupta DS, Griem ML. Pacemaker failure resulting from radiation damage. Radiology. 1983; 148: 257-8.

[3] Calfee RV. Therapeutic radiation and pacemakers. Pacing Clin Electrophysiol. 1982; 5: 160-1.
[4] Lee RW, Huang SK, Mechling E, Bazgan I. Runaway atrioventricular sequential pacemaker after radiation therapy. Am J Med. 1986; 81: 883-6.

[5] Last A. Radiotherapy in patients with cardiac pacemakers. Br J Radiol. 1998; 71: 4-10.

[6] Pinski SL, Trohman RG. Interference with cardiac pacing. Cardiol Clin. 2000; 18: 219-39.

[7] Wilkoff BL, Cook JR, Epstein AE, Greene HL, Hallstrom AP, Hsia H, et al. Dual-chamber pacing or ventricular backup pacing in patients with an implantable defibrillator: the Dual Chamber and VVI Implantable Defibrillator (DAVID) Trial. JAMA. 2002; 288: 3115-23.

[8] Marbach JR, Meoz-Mendez RT, Huffman JK, Hudgins PT, Almond PR. The effects of cardiac pacemakers of ionizing radiation and electromagnetic interference from radiotherapy machines. Int $\mathrm{J}$ Radiat Oncol Biol Phys. 1978; 4: 1055-8.

[9] Ziółkowska E, Zarzycka M, Wiśniewski T, Windorbska W, Meller A. Zastosowanie radioterapii u chorych ze stymulatorem serca Część II. Standardy napromieniania pacjentów ze stymulatorem serca - przegląd literatury. Wspolczesna Onkologia. 2008; 12: 400-5.

[10]Ziółkowska. E., Wiśniewski T, Białas M, Balcerzak M, Woźniak-Wiśniewska A, Sinkiewicz W. Radiation therapy with Implanted Cardiac Pacemaker or Implantable Cardioverter Defibrillator: poposed precautions. Oncology and Radioherapy. 2012; 3: 40-8.

[11] Hudson F, Coulshed D, D'Souza E, Baker C. Effect of radiation therapy on the latest generation of pacemakers and implantable cardioverter defibrillators: A systematic review. J Med Imaging Radiat Oncol. 2010; 54: 53-61.

[12] Sznajder R, Kargul W. Nowe algorytmy stymulacyjne w migotaniu przedsionków _ stymulatory antyarytmiczne. Folia Cardiol 2005; 12:

[13] Marbach JR, Sontag MR, Van Dyk J, Wolbarst AB. Management of radiation oncology patients with implanted cardiac pacemakers: report of AAPM Task Group No. 34. American Association of Physicists in Medicine. Med Phys. 1994; 21: 85-90.

[14] Uiterwaal GJ, Springorum BGF, Scheepers E, de Ruiter GS, Hurkmans CW. Interference detection in implantable defibrillators induced by therapeutic radiation therapy. Neth Heart J. 2006; 14: 330-4.

\section{Author Profile}

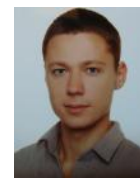

Mieszko Białas MD, Department of Radiotherapy, Prof. F. Łukaszczyk Memorial Oncology Center, Bydgoszcz, Poland. 